\title{
ON THE OVERCONVERGENCE OF CERTAIN SEQUENCES OF RATIONAL FUNCTIONS OF BEST APPROXIMATION.
}

BY

\author{
J. L. WALSH
}

of Cambridge, Mass.

I. Introduction. For many purposes, arbitrary rational functions are more useful in approximating to given analytic functions of a complex variable than are polynomials. For instance it is shown by Runge in his classical paper on approximation by polynomials ${ }^{1}$ that a function $f(z)$ analytic in a closed region of the $z$-plane bounded by a finite number of non-intersecting Jordan curves can be uniformly approximated in that region as closely as desired by a rational function of $z .^{2}$ Such approximation by a polynomial may not be possible. It is the purpose of the present paper to show that in the study of two other phases of approximation it may also be more advantageous to use general rational functions than polynomials, namely I) degree of approximation, that is, asymptotic properties of the measure of approximation of the sequence of functions of best approximation, and 2) overconvergence, the phenomenon that a sequence of functions approximating a given function in a given region frequently converges to that given function (or its analytic extension) not merely in the given region but also in a larger region containing the given region in its interior. The term overconvergence has recently been used by Ostrowski in a somewhat different connection.

A rational function of the form

1 Acta mathematica vol. 6 (I 885), pp. 229-244.

2 For more detailed results, compare Walsh, Mathematische Annalen vol. 96 (I926), pp. $437^{-}-450$ and Transactions of the American Mathematical Society vol. 3I (I929), pp. 477-502. 


$$
\frac{a_{0} z^{n}+a_{1} z^{n-1}+\cdots+a_{n}}{b_{0} z^{n}+b_{1} z^{n-1}+\cdots+b_{n}}
$$

where the denominator does not vanish identically, is said to be of degree $n$.

We shall deal with the entire plane of the complex variable $z$, closed by the adjunction of a single point at infinity. The derivative, or more explicitly the first derivative of an arbitrary point set $E$ is the set $E^{\prime}$ composed of the limit points of $E$. The second derivative of $E$ is the first derivative $E^{\prime \prime}$ of $E^{\prime}$, and in general the $k$-th derivative $E^{(k)}$ of $E$ is similarly defined as the first derivative of the $(k-1)$-st derivative of $E$. The principal result of the present paper is

Theorem I. Suppose $f(z)$ is an analytic function of $z$ whose singularities form $a$ set $E$ one of whose derivatives $E^{(k)}$ is empty. Suppose $C$ is a closed point set with no point in common with $E$. Then a sequence of rational functions $r_{n}(z)$ of respective degrees $n$ of best approximation to $f(z)$ on $C$ such that the poles of $r_{n}(z)$ lie in $\boldsymbol{E}$, converges to the function $f(z)$ over the entire plane except on the set $E$. The convergence is uniform on any closed point set containing no point of $E$, and on any such point set the convergence is better than that of any geometric series.

The term best approximation deserves some explanation. There are various measures of approximation of the function $r_{n}(z)$ to the given function $f(z)$ defined in a region $C$, for instance $\max || f(z)-r_{n}(z) \mid, z$ on $\left.C\right], \cdot \int\left|f(z)-r_{n}(z)\right|^{2}|d z|$ taken over the boundary of $C$, or $\iint\left|f(z)-r_{n}(z)\right|^{2} d S$ taken over the area of $C$. Let us consider a particular measure of approximation and a particular value of $n$, and call admissible any rational function of degree $n$ whose poles lie in $E$. Then a rational function $r_{n}(z)$ of degree $n$ of best approximation to $f(z)$ on $C$ such that the poles of $r_{n}(z)$ lie in $E$ is that admissible function $r_{n}(z)$ or one of those admissible functions whose measure of approximation to $f(z)$ on $C$ is less than the measure of approximation to $f(z)$ on $C$ of any other admissible function. It is not obvious but can be shown without great difficulty that such a function of best approximation always exists, for the various measures of approximation that we shall use, although it need not be unique. ${ }^{1}$

\footnotetext{
1 See Walsh, Transactions of the American Mathematical Society vol. 33 (I93I).

The existence af a function of best approximation depends essentially on the closure of the set $E$.

If $E$ contains but a finite number of points, there are for a given $n$ but a finite number of possible distributions of the orders of the poles of $r_{n}(z)$ among the points of $E$. For each such
} 
In Theorem $I$ the set $C$ may be I) any closed point set not a single point whose complement is simply connected, approximation being measured in the sense of Tchebycheff: 2) any closed set not a single point whose complement is simply connected, approximation being measured by integration over the circle $\gamma:|w|=I$ when the complement of $C$ is mapped onto the exterior of $\gamma$; 3) any limited closed set $C$ whose boundary is a rectifiable Jordan arc or curve, or more generally any limited set $C$ whose boundary $C^{\prime}$ is of positive linear measure and whose complement is simply connected, approximation being measured by a line integral over $C^{\prime} ;$ 4) any simply connected region, approximation being measured by integration on the circle $\gamma:|w|==I$ when $C$ is mapped onto the interior of $\gamma$; 5) any region or point set. with at least one interior point and having positive area, approximation being measured by a double integral over $C{ }^{1}$

By approximation in the sense of Tchebycheff we understand that the measure of approximation of $r_{n}(z)$ to a given function $f(z)$ on a point set $C$ is

$$
\max \left[\left|f(z)-v_{n}(z)\right|, z \text { on } C\right]
$$

In this measure of approximation it is a slight generalization to insert a weight or norm function $n(z)$ positive and continuous on $C$ and to use as the measure of approximation

$$
\max \left[n(z)\left|f(z)-r_{n}(z)\right|, z \text { on } C\right]
$$

This introduction of a norm function presents no difficulty, and for the sake of simplicity we do not make the introduction for the Tchebycheff measure of approximation. We do introduce a norm function, however, for the integral measures of approximation 2)-5).

The measures of approximation I)-5) have recently been used by the present writer in the study of approximation to given analytic functions by polynomials, ${ }^{2}$ and results analogous to Theorem I have been established. It is to be noticed that in Theorem I the case that $f(z)$ is an entire function leads

distribution there is (loc. cit.) but a single rational function of best approximation, and hence independently of this distribution there are but a finite number of functions $r_{n}(z)$ of best approximation.

1 The reader may notice from the discussion which follows that in all of these cases the reasoning we give is valid or can be modified so as to be valid even if the complement of $C$ is finitely multiply connected, provided that $C$ contains no isolated point.

2 Transactions of the American Mathematical Society, vol. 32 (1930), pp. 794-8I6, and vol. 33 (193I), pp. $370-388$. 
precisely to the approximation of $f(z)$ by polynomials, which case has been treated with others in the papers just mentioned.

The results just referred to are perhaps worth stating in detail so that they can be compared with Theorem I. Special cases of these results are naturally due to various other writers; we shall have occasion later to mention the special case due to $\mathrm{S}$. Bernstein. Let $C$ be an arbitrary limited closed point set of the $z$-plane and denote by $I$ the set of all points each of which can be joined to the point at infinity by a broken line which does not meet $C$. We suppose $D$ to be simply connected. Let the function $w=\varphi(z)$ map $D$ onto the exterior of $|w|=I$ so that the points at infinity correspond to each other and denote by $C_{R}$ the curve $|\varphi(z)|=R>\mathrm{I}$ in the $z$-plane, namely the image of the circle $|w|=R$. If the function $f(z)$ is analytic interior to $C_{R}$ but has a singularity on $C_{R}$, the sequence of polynomials $\pi_{n}(z)$ of best approximation to $f(z)$ on $C$ (measured in any one of the ways I) - 5) provided that in 5) the point set $C$ is a closed region], converges to $f(z)$ for $z$ interior to $C_{R}$, uniformly for $z$ on any closed point set interior to $C_{R}$, and converges uniformly in no region containing in its interior a point of $C_{R}$. If $R_{1}<R$ and if the measure of approximation $\mu_{n}$ involves the $p$-th power of $\left|f(z)-\pi_{n}(z)\right|, p>0$, then the inequality

$$
\mu_{n} \leqq \frac{M}{R_{1}^{n p}}, \quad n=1,2, \ldots
$$

is valid, where $M$ depends on $R_{1}$ but not on $n$, but this inequality is valid for no choice of $R_{1}>R$.

A somewhat trivial but nevertheless illuminating illustration of the difference between polynomials and more general rational functions when used for the approximation of a given function, occurs for a function $f(z)$, approximated in the sense of least squares on the unit circle $C:|z|=I$ and having a single singularity in the plane, namely at the point $z=a$ whose modulus is greater than unity. The sequence of polynomials of best approximation to $f(z)$ on $C$ in the sense of least squares is the sequence of partial sums of the Taylor development of $f(z)$ at the origin. This sequence $\left\{\pi_{n}(z)\right\}$ converges in such a way that we have

$$
\int_{C}\left|f(z)-\pi_{n}(z)\right|^{2}|d z| \leqq \frac{M}{R^{2 n}}, \quad n=\mathrm{I}, 2, \ldots,
$$

where $R$ is an arbitrary number less than $|a|$, but this inequality holds for no 
choice of $M$ when $R$ is greater than $|a|$. The sequence $\left\{\pi_{n}(z)\right\}$ converges for $|z|<|a|$ and diverges for $|z|>|a|$.

On the other hand, if we study the best approximation to $f(z)$ on $C$ in the sense of least squares by rational functions $r_{n}(z)$ of respective degrees $n$ whose poles lie in the point $z=a$, the inequality

$$
\int_{\partial}\left|f(z)-r_{n}(z)\right|^{2}|d z| \leqq \frac{M}{R^{2 n}}, \quad n=\mathrm{I}, 2, \ldots
$$

is satisfied for an arbitrary $R$, provided that a suitable $M$ (depending naturally on $R$ ) is chosen. This fact is easily proved for itself by help of the transformation $w=(1-\bar{a} z) /(z-a)$ and indeed follows from Theorem $\mathrm{I}$, as does the fact that the corresponding sequence $\left\{r_{n}(z)\right\}$ converges to the sum $f(z)$ at every point of the plane other than $z=a$. Thus the degree of approximation is not so great for approximation to $f(z)$ on $C$ by polynomials as for approximation by rational functions with poles in $z=a$, and in the latter case the region of convergence is also greater.

Theorem $\mathrm{I}$ is true in the trivial case that $C$ is the entire plane, for in this case $f(z)$ must be a constant and all the approximating rational functions $r_{n}(z)$ are this same constant. Approximation on $C$ can be measured by either of the methods I) or 5). Henceforth this trivial case is excluded.

2. Degree of Approximation. A preliminary theorem which we shall apply is

Theorem II. Suppose $f(z)$ is an analytic function of $z$ whose singularities form a set $E$ one of whose derivatives $E^{(k)}$ is empty. Suppose $C$ is a closed point set with no point in common with $E$. Then there exists a sequence of rational functions $r_{n}(z)$ of respective degrees $n$ whose poles lie on $E$ such that for an arbitrary $R$ we have

$$
\left|f(z)-r_{n}(z)\right| \leqq \frac{M}{R^{n}}, \quad z \text { on } C
$$

where $M$ depends on $R$ but not on $n$.

We prove Theorem II first for the case that $E^{\prime}$ is empty, so that $E$ consists of a finite number of points $A_{1}, A_{2}, \ldots, A_{p}$.

The function $f(z)$ can be expressed as the sum of $v$ functions, each analytic on the entire extended plane except in a point $A_{k}$. In fact, let us assume that 
$A_{1}$ is the point at infinity; this is no restriction of generality. Then Cauchy's integral

$$
f(z)=\sum_{k=1}^{v} \frac{\mathrm{I}}{2 \pi i} \int_{\gamma_{k}} \frac{f(t) d t}{t-z}
$$

gives this expression directly, if $\gamma_{1}$ is a circle containing $A_{2}, A_{3}, \ldots, A_{v}$, and $\gamma_{k}(k>\mathrm{I})$ is a circle about the point $A_{k}$ but containing no other point $A_{j}$. Equation (2. I) is valid if $z$ lies in the region bounded by these $v$ circles, integration being taken in the positive sense with respect to this region. The integrals in $(2.1)$ are all independent of the particular circles $\gamma_{k}$ chosen, provided merely that the circle $\gamma_{1}$ is sufficiently large and the other circles are sufficiently small; each integral defines a function analytic over the entire extended plane except at a point $A_{k}$. Let us introduce the notation

$$
f_{k}(z)=\frac{\mathrm{I}}{2 \pi i} \int_{i k} \frac{f(t) d t}{t-z},
$$

it being understood that the circle $\gamma_{k}$ is so chosen as to separate $z$ and $A_{k}$, but not to separate $z$ and any other point $A_{j}$. The function $f_{k}(z)$ is thus defined and analytic at every point of the extended plane except at $A_{k}$.

The function $f_{k}(z)$ can be uniformly approximated in the sense of Tchebycheff on the point set $C$ by a sequence of rational functions $r_{n}^{(k)}(z)$ of respective degrees $n$ whose poles lie in $A_{k}$ and such that we have

$$
\left|f_{k}(z)-r_{n}^{(k)}(z)\right| \leqq \frac{\boldsymbol{M}_{k}}{\boldsymbol{R}_{k}^{n}}, \quad z \text { on } C,
$$

where $R_{k}>\mathrm{I}$ is arbitrary and $M_{k}$ depends on $R_{k}$. In fact, if we transform $A_{k}$ into the point at infinity by a linear transformation of the complex variable, the successive convergents of degree $n$ of the Taylor development of the transformed $f_{k}(z)$, about the new origin yield by transformation back to the original situation a suitable set of functions $r_{n}^{(k)}(z)$. The rational function

$$
r_{n n}(z)=\sum_{k=1}^{v} r_{n}^{(k)}(z)
$$

may be considered of degree $\nu n$, so we may write by addition of inequalities $(2 \cdot 3)$ 


$$
\left|f(z)-r_{v n}(z)\right| \leqq \frac{M^{\prime}}{R_{1}^{n}}, \quad z \text { on } C
$$

where all the numbers $R_{k}$ are chosen the same and $M^{\prime}$ is the sum of the $M_{k}$. This inequality does not yet hold for rational functions of all degrees, but we may write

$$
\left|f(z)-r_{m}(z)\right| \leqq \frac{M}{R^{m}}, z \text { on } C
$$

where we set $R=R_{1}^{1 / \nu}$, where we set

$$
r_{m}(z)=r_{v n}(z)
$$

$\boldsymbol{\nu} n$ being the smallest multiple of $\boldsymbol{\nu}$ not less than $m$, and where we have $M=M^{\prime} R_{1}$. Inequality (2.4) thus holds for all $m$, where $r_{m}(z)$ is a rational function of degree $m$ and where $R>\mathrm{I}$ is arbitrary.

This completes the proof of Theorem II in the case that $E^{\prime}$ is empty. Let us treat next the case that $E^{\prime \prime}$ is empty, so that $E^{\prime}$ consists of a finite number of points $A_{1}, A_{2}, \ldots, A_{v}$; we assume that $A_{1}$ is the point at infinity. Let $R>$ r be given. Let $\gamma_{1}$ be a large circle containing $A_{2}, A_{3}, \ldots, A_{v}$ in its interior and let $\gamma_{2}, \gamma_{3}, \ldots, \gamma_{v}$ be small circles about the points $A_{2}, A_{3}, \ldots, A_{v}$ respectively. Let $\delta$ denote half the maximum diameter of $C$. Then the radius of $\gamma_{1}$ is to be chosen larger than $\delta R^{v+1}$. The radius of $\gamma_{2}$ is to be chosen so small that when $A_{2}$ is transformed to infinity by a linear transformation of the complex variable the radius of the corresponding circle (transform of $\gamma_{2}$ ) is larger than the product of $R^{\nu+1}$ by half the maximum diameter of the transform of $C$. The radii of $\gamma_{3}, \gamma_{4}, \ldots, \gamma_{v}$ are to be chosen correspondingly. None of these circles $\gamma_{i}$ shall pass through a singularity of $f(z)$.

Cauchy's integral

$$
f(z)=\sum_{k=1}^{v+1} \frac{1}{2 \pi i} \int_{\gamma_{k}} \frac{f(t) d t}{t-z}, z \text { on } C,
$$

where $\gamma_{v+1}$ is an arbitrary curve or curves separating $C$ from none of the circles $\gamma_{1}, \ldots, \gamma_{\nu}$ but separating $C$ from all the singular points of $f(z)$ interior to $\gamma_{1}$ and exterior to $\gamma_{2}, \gamma_{3}, \ldots, \gamma_{\nu}$, expresses $f(z)$ as the sum of $v+1$ functions which are analytic respectively interior to $\gamma_{1}$, exterior to $\gamma_{2}, \gamma_{3}, \ldots, \gamma_{v}$, and exterior to $\gamma_{v+1}$. The function

$$
\frac{\mathrm{I}}{2 \pi i} \int_{\gamma_{\nu+1}} \frac{f(t) d t}{t-z}
$$

53-31104. Acta mathematica. 57. Imprimé le 3 septembre 1931. 
is independent of the particular curve or curves $\gamma_{v+1}$ chosen, and the only singularities of this function are the points of $E$ not exterior to $\gamma_{1}$ or interior to $\gamma_{2}, \gamma_{3}, \ldots, \gamma_{\nu}$.

There exist rational functions $r_{n}^{(i)}(z)$ of respective degrees $n$ such that we have

$$
\left|\frac{1}{2 \pi i} \int_{\gamma_{i}} \frac{f(t) d t}{t-z}-r_{n}^{(i)}(z)\right| \leqq \frac{M^{(i)}}{R^{n(v+1)}}, z \text { on } C, \quad i=\mathrm{I}, 2, \ldots, \nu ;
$$

in fact the rational function $r_{n}^{(i)}(z)$ may be chosen so as to have all its poles in the point $A_{i}$, and the function $r_{n}^{(i)}(z)$ may be chosen as the sum of the first $n+\mathrm{I}$ terms of the Taylor development of the function approximated, about a suitable point, when $A_{i}$ is transformed to infinity by a suitable linear transformation of the complex variable. It follows from the particular choice of the circles $\gamma_{i}$ that the inequality (2.5) will be satisfied by these particular rational functions. The function

$$
\frac{1}{2 \pi i} \int_{\gamma_{v+1}} \frac{f(t) d t}{t-z}
$$

has as its only singularities in the plane the points of $E$ not exterior to $\gamma_{1}$ or interior to $\gamma_{2}, \gamma_{3}, \ldots, \gamma_{v}$, and these singularities of this function are finite in number. Then by the part of Theorem II already established (i. e. $E^{\prime}$ empty), there exist rational functions $r_{n}^{(v+1)}(z)$ of respective degrees $n$ such that we have

If we set

$$
\left|\frac{1}{2 \pi i} \int_{\gamma_{v+1}} \frac{f(t) d t}{t-z}-r_{n}^{(v+1)}(z)\right| \leqq \frac{M^{(v+1)}}{R^{n(v+1)}}, \quad z \text { on } C .
$$

$$
r_{(v+1) n}(z)=\sum_{k=1}^{v+1} r_{n}^{(k)}(z)
$$

we have a rational function of degree $(\nu+1) n$ with the property

$$
\left|f(z)-r_{(v+1) n}(z)\right| \leqq \frac{M_{1}}{R^{(v+1) n}}, \quad z \text { on } C .
$$

We now make the definition

$$
r_{m}(z)=r_{(v+1) n}(z)
$$

where $(\nu+\mathrm{I}) n$ is the smallest multiple of $v$ not less than $m$, so by setting $M=M_{1} R^{v+1}$ we have the inequality 


$$
\left|f(z)-r_{m}(z)\right| \leqq \frac{M}{R^{m}}, \quad z \text { on } C,
$$

which holds for all values of $m, R>\mathrm{I}$, and Theorem II is established in the case that $E^{\prime \prime}$ is empty.

A formal proof of Theorem II in the general case that $E^{(k)}$ is empty follows directly the proof just given, by the use of mathematical induction, and the details are left to the reader.

In the present paper we are primarily concerned with the rational functions of degree $n$ of best approximation. By Theorem II there exists some sequence of rational functions $r_{n}(z)$ of respective degrees $n$ whose poles lie on the set $E$ such that (2.4) is satisfied. It follows directly that the same inequality must be valid for the sequence of rational functions $r_{n}(z)$ of best approximation in the sense of Tchebycheff whose poles lie on $E$.

3. A Theorem on Overconvergence. Another preliminary theorem which we shall have occasion to use is

Theorem III. If the sequence of rational functions $r_{n}(z)$ of respective degrees $n$ converges in a region $C^{\prime}$ (containing no limit point of poles of the $r_{n}(z)$ ) in such a way that we have for every $R$

$$
\mid f(z)-r_{n}(z) \leqq \frac{M}{R^{n}}, \quad z \text { in } C^{\prime},
$$

where $M$ depends on $R$ but not on $n$, then the sequence $\left\{r_{n}(z)\right\}$ converges and $f(z)$ is analytic at every point of the extended plane except the limit points of poles of the functions $r_{n}(z)$ and except points separated from $C^{\prime}$ by such limit points. Convergence is uniform on any closed region $C^{\prime \prime}$ containing no such limit point, and for $z$ on $C^{\prime \prime}$ an inequality of form (3. I) holds for an arbitrary $R$ provided that $M$ (depending on $R$ ) is suitably chosen.

In the proof of Theorem III we need to apply a lemma of which a special case was first used by S. Bernstein. The proof of the present lemma is inspired directly by the proof of Bernstein's special case given by Marcel Riesz in a letter to Mittag-Leffler. ${ }^{1}$ The entire discussion of the present paper is analogous to Bernstein's discussion in which his lemma was proved. His chief result in this connection is that if a function $f(z)$ is analytic on and within the ellipse with foci $\mathrm{I}$ and $-\mathrm{I}$ and semi-axes $a$ and $b$, then there exist polynomials $p_{n}(z)$ of

\footnotetext{
' Acta mathematica vol. 40 (I9I6), pp. $337-347$.
} 
respective degrees $n$ such that we have

$$
\left|f(z)-p_{n}(z)\right| \leqq \frac{M}{\varrho^{n}}, \quad-\mathrm{I} \leqq z \leqq+\mathrm{I}, \quad \varrho=a+b .
$$

Reciprocally, if there exist polynomials $p_{n}(z)$ of respective degrees $n$ such that (3.2) is satisfied for $-\mathrm{I} \leqq z \leqq+\mathrm{I}$, for a certain value of $\varrho$, then the function $f(z)$ is analytic interior to the ellipse described. Our Theorem II is the analogue of the first part of Bernstein's theorem, and our Theorem III is the analogue of the second part of that theorem.

The following lemma has already been established elsewhere, ${ }^{1}$ although in a slightly less general form, but the proof is simple and typical of other proofs to be given, and so will be repeated.

Lemma I. Let $\Gamma$ be an arbitrary closed limited point set of the $z$-plane whose complement is simply connected, and denote by $w=\Phi(z), z=\Psi(w)$, a function which maps the complement of $\Gamma$ onto the exterior of the unit circle $\gamma$ in the w-plane so that the two points at infinity correspond to each other. Let $\Gamma_{R}$ denote the curve $|\Phi(z)|=R>I$ in the z-plane, the transform of the circle $|w|=R$. If $P(z)$ is a rational function of degree $n$ whose poles lie exterior to $\Gamma_{\rho}, \varrho>1$, and if we have

then we have likewise

$$
|P(z)| \leqq L, \quad z \text { on } \Gamma
$$

$$
|P(z)| \leqq L\left(\frac{\varrho R_{1}-\mathrm{I}}{\varrho-\bar{R}_{1}}\right)^{n}, \quad z \text { on } \Gamma_{R_{1}}, \quad R_{1}<\varrho .
$$

In the statement of Lemma I we have, as a matter of convenience, required that $\Gamma$ should be limited and that in the conformal mapping the point at infinity in the $z$-plane should be transformed into the point at infinity in the $w$-plane. The result can naturally be phrased in terms of an arbitrary closed point set $\Gamma$, where in the conformal mapping an arbitrary point of the complement of $\Gamma$ is transformed into the point at infinity in the $w$-plane.

The function $P[\Psi(w)]$ has at most $n$ poles for $|w| \geqq \mathrm{I}$ and these all lie exterior to $|w|=\varrho$. For convenience in exposition we suppose that there are precisely $n$ poles $\alpha_{1}, \alpha_{2}, \ldots, \alpha_{n}$, not necessarily all distinct, and that none of them lies at infinity. If there are less than $n$ poles, or if infinity is also a pole, p. 842 .

${ }^{1}$ Walsh, Transactions of the American Mathematical Society, vol 30 (1928), pp. 838-847; 
there are only obvious modifications to be made in the discussion. The function

$$
\pi(w)=P[\Psi(w)] \frac{\left(w-\alpha_{1}\right)\left(w-\alpha_{2}\right) \cdots\left(w-\alpha_{n}\right)}{\left(\mathrm{I}-\bar{\alpha}_{1} w\right)\left(\mathrm{I}-\bar{\alpha}_{2} w\right) \cdots\left(\mathrm{I}-\bar{\alpha}_{n} w\right)}
$$

is analytic for $|w|>1$. When $w(|w|>1)$ approaches $\gamma, z$ approches $C$, and all limiting values of $|P[\Psi(w)]|$ are less than or equal to $L$; the function $\left(w-\alpha_{i}\right) /\left(\mathrm{I}-\bar{\alpha}_{i} w\right)$ is continuous and has the modulus unity on $\gamma$, from which it follows that the limiting values of $|\pi(w)|$ for $w$ approaching $\gamma(|w|>1)$ are not greater than $L$. Then we have

$$
|\pi(w)| \leqq L
$$

for $|w|>1$, since the function $|\pi(w)|$ can have no maximum for $|w|>1$.

The transformation $\zeta=\left(w-\alpha_{i}\right) /\left(\mathrm{I}-\bar{\alpha}_{i} w\right)$ transforms $|w|=R_{1}$ into the circle $\left|\left(\zeta+\alpha_{i}\right) /\left(\mathrm{I}+\bar{\alpha}_{i} \zeta\right)\right|=R_{1}$, so we have

$$
|\zeta| \geqq \frac{\left|\alpha_{i}\right|-R_{1}}{R_{1}\left|\alpha_{i}\right|-\mathrm{I}} \geqq \frac{\varrho-R_{1}}{R_{1} \varrho-\mathrm{I}} \quad \text { for }|w|=R_{1}<\varrho .
$$

Thus we find from (3.3) and (3.4),

$$
|P[\Psi(w)]| \leqq L \prod_{i=1}^{n}\left|\frac{1-\bar{\alpha}_{i} w}{w-\alpha_{i}}\right| \leqq L\left(\frac{R_{1} \varrho-\mathrm{I}}{\varrho-R_{1}}\right)^{n}
$$

for $|w|=R_{1}<\varrho$, and Lemma $I$ is established.

Lemma $I$ in the form in which we have considered it, is not expressed so as to be invariant under all linear transformations of the complex variable. That it to say, a suitable linear transformation yields a new result. One way in which we shall apply Lemma $I$ is in proving the following remark:

If the sequence of rational functions $P_{n}(z)$ of respective degrees $n$ satisfy the inequality

$$
\left|P_{n}(z)\right| \leqq \frac{M_{1}}{R_{1}^{n}}, \quad z \text { in a circular region } K
$$

for every value of $R_{1}$, where $M_{1}$ depends on $R_{1}$, and if the circular region $K^{\prime}$ contains $K$ but contains on or within it no limit point of poles of the functions $P_{n}(z)$, then the inequality 


$$
\left|P_{n}(z)\right| \leqq \frac{M_{9}}{R_{9}^{n}}, \quad z \text { in } K^{\prime}
$$

is satisfied for every value of $R_{2}$, where $M_{2}$ depends on $R_{2}$.

In the sense here considered, a circular region is the closed interior or exterior of a circle, or a closed half-plane. The proof of the remark follows directly from the lemma, by transforming the given circular regions $K$ and $K^{\prime}$ into two regions bounded by concentric circles of respective radii $I$ and $\varrho_{1}>$ I. This transformation is naturally to be a linear transformation of the complex variable, and to prove the remark we need merely set

$$
\frac{\mathrm{I}}{R_{2}}=\frac{\mathrm{I}}{R_{1}} \frac{\varrho \varrho_{1}-\mathrm{I}}{\varrho-\varrho_{1}}, \quad M_{1}=M_{2}
$$

where the circle concentric with $K$ and $K^{\prime}$ of radius $\varrho$ contains $K^{\prime}$ but contains no limit point of poles of the functions $P_{n}(z)$.

Theorem III follows directly from Lemma I and from the remark just made. From the inequalities

$$
\begin{gathered}
\left|f(z)-r_{n-1}(z)\right| \leqq \frac{M}{R^{n-1}}, \quad z \text { in } C^{\prime} \\
\left|f(z)-r_{n}(z)\right| \leqq \frac{M}{R^{n}}, \quad z \text { in } C^{\prime}
\end{gathered}
$$

we derive

$$
\left|r_{n}(z)-r_{n-1}(z)\right| \leqq \frac{N}{R^{n}}, \quad z \text { in } C^{\prime}
$$

where $N=M(\mathrm{I}+R)$. The function $r_{n}(z)-r_{n-1}(z)$ is rational of degree $2 n-\mathrm{I}$, so if the point set $C^{\prime}$ is limited, and this situation can be reached by a linear transformation, we obtain from the lemma

$$
\left|r_{n}(z)-r_{n-1}(z)\right| \leqq \frac{N}{R^{n}}\left(\frac{\varrho R_{1}-1}{\varrho-R_{1}}\right)^{2 n-1}, \quad z \text { on } C_{R_{1}}^{\prime}, \quad R_{1}<\varrho
$$

for $n$ sufficiently large, where $C_{\rho}^{\prime}$ contains on or within it no limit point of poles of the functions $r_{n}(z)$, and it is to be remembered that $R$ is arbitrary. It follows that the sequence $\left\{r_{n}(z)\right\}$ converges interior to any curve $C_{R_{1}}^{\prime}$ which contains on or within it no limit point of poles of that sequence, and that in any such curve $C_{R_{1}}^{\prime}$ the inequality 


$$
\left|r_{n}(z)-r_{n-1}(z)\right| \leqq \frac{M}{R^{n}}
$$

holds for an arbitrary choice of $R$.

By a method entirely analogous to that of analytic extension ${ }^{1}$ it can now be shown from the remark following Lemma $I$ that this same inequality holds in the region $C^{\prime \prime}$ of Theorem III. Inequality (3.5), holding in some region $C_{R_{1}}^{\prime}$, holds also in any circular region containing no limit point of poles of the $r_{n}(z)$ but having a subregion in common with $C_{R_{1}}^{\prime}$. The process of extending step by step the domain of known validity of (3.5) can be stopped only by limit points of poles of the $r_{n}(z)$, and any point set such as the $C^{\prime \prime}$ prescribed in Theorem III can be included in this domain by a finite number of steps. The uniform convergence on $C^{\prime \prime}$ of the sequence $\left\{r_{n}(z)\right\}$ follows directly from (3.5), and the identity of the limit function with $f(z)$ (or its analytic extension) follows from (3. I) for $z$ in $C^{\prime}$ and hence for $z$ on $C^{\prime \prime}$. There is no difficulty in deriving (3. I) for $z$ on $C^{\prime \prime}$ from (3.5) for $z$ on $C^{\prime \prime}$, so the proof of Theorem III is complete.

We have now a proof of Theorem I in the case I), that approximation is measured in the sense of Tchebycheff. Inequality (3. I) holds for the sequence of rational functions of best approximation whose poles lie in $E$, as we have already indicated, and the conclusion of Theorem I follows from Theorem III.

4. Approximation measured after Conformal Mapping. We now take up the measure 2) of approximation to $f(z)$ on $C$, that $C$ is an arbitrary closed set not a single point whose complement is simply connected, and approximation is measured in the sense of weighted $p$-th powers $(p>0)$ by integration on the circle $\gamma:|w|=\mathrm{I}$ when the complement of $C$ is mapped onto the exterior of $\gamma$. This measure of approximation naturally depends on the particular point $O^{\prime}$ of the complement of $C$ chosen to correspond to the point at infinity in the $w$-plane, but the problem of best approximation for a particular choice of $O^{\prime}$ with a particular choice of the norm function $n(w)$ is equivalent to the problem of best approximation for an arbitrary choice of $O^{\prime}$ with a suitable norm function $n(w)$. In the present paper we suppose $O^{\prime}$, once determined, to be fixed. A similar

${ }^{1}$ Ostrowski has indicated the close analogy between analytic extension and the study of regions of convergence of certain series. See for instance Abhandlungen aus dem Mathematischen Seminar der Hamburgischen Universität, Vol. I (1922), pp. 327-350. 
remark applies to the norm function and to the particular map chosen for our other measures of approximation involving conformal mapping.

We shall need the following lemma:

Lemma II. Let $C$ be an arbitrary limited closed point set of the z-plane, not a single point, whose complement is simply connected, and denote by $w=\boldsymbol{\Phi}(z)$, $z=\Psi(w)$ a function which maps the complement of $C$ onto the exterior of the unit cirele $\gamma$ in the w-plane so that the points at infinity correspond to each other. Let $C_{R}$ denote the curve $|\Phi(z)|=R>\mathrm{I}$ in the z-plane. If $P(z)$ is a rational function of degree $n$ whose poles lie exterior to $C_{\varrho}, \varrho>\mathrm{I}$, and if we have

$$
\int_{\gamma}|P(z)|^{p}|d x| \leqq L^{p}, \quad p>0,
$$

then we have likewise

$$
|P(z)| \leqq L L^{\prime}\left(\frac{\varrho R_{1}-1}{\varrho-R_{1}}\right)^{n}, \quad z \text { on } C_{R_{1}}, \quad R_{1}<\varrho,
$$

where $L_{1}$ depends on $R_{1}$ but not on $P(z)$.

Properly speaking, the function $P[\Psi(w)]$ is not defined on $\gamma$, and therefore the use of the integral (4. I) requires some explanation. The function $\Psi(w) / w$ is analytic and uniformly limited for $|w|>1$, and therefore by Fatou's theorem ${ }^{1}$ this function and hence the function $\Psi(w)$ approaches a limit almost everywhere on $\gamma$ when $w$ remains exterior to $\gamma$ and approaches $\gamma$ along a radius. When $w$ approaches $\gamma$, the function $z=\Psi(w)$ approaches a boundary point of $C$ and hence $P[\Psi(w)]$ approaches a limit. It is these values of $P[\Psi(w)]$, which therefore exist almost everywhere on $\gamma$, that are intended to be used in the integral in (4. I). A similar fact holds for the other measures of approximation that we shall use which depend on conformal mapping.

The proof of Lemma II is quite similar to the proof of Lemma I. The function $P[\Psi(w)]$ has at most $n$ poles for $|w| \geqq \mathrm{I}$, and these all lie exterior to $|w|=\varrho$. For convenience in exposition we shall suppose that there are precisely $n$ poles $\alpha_{1}, \alpha_{2}, \ldots, \alpha_{n}$, not necessarily all distinct, and that none lies at infinity. If there are less than $n$ poles, or if infinity is also a pole, there are only obvious modifications to be made in the discussion. In the latter case, for instance, we consider in the right-hand member of (4.2) the function found by taking the

${ }^{1}$ Acta mathematica, vol. 30 (1906), pp. $335-400$. 
limit as one or more of the $\alpha_{i}$ become infinite. Similarly let $\beta_{1}, \beta_{2}, \ldots, \beta_{n}$ denote the zeros of $P[\Psi(w)]$ exterior to $\gamma$. The function

$$
\text { (4. 2) } \pi(w)=P[\Psi(w)] \frac{\left(w-\alpha_{1}\right)\left(w-\alpha_{2}\right) \cdots\left(w-\alpha_{n}\right)\left(\mathrm{I}-\bar{\beta}_{1} w\right)\left(\mathrm{I}-\bar{\beta}_{2} w\right) \cdots\left(\mathrm{I}-\vec{\beta}_{n} w\right)}{\left(\mathrm{I}-\bar{\alpha}_{1} w\right)\left(\mathrm{I}-\bar{\alpha}_{2} w\right) \cdots\left(\mathrm{I}-\bar{\alpha}_{n} w\right)\left(w-\beta_{1}\right)\left(w-\beta_{2}\right) \cdots\left(w-\beta_{n}\right)}
$$

is analytic and different from zero for $|w|>\mathrm{I}$, and on $\gamma:|w|=\mathrm{I}$ we have for the values taken on by normal approach to $\gamma$,

$$
|\pi(w)|=|P[\Psi(w)]|
$$

The hypothesis of Lemma II is therefore

$$
\int_{\gamma}|\pi(w)|^{p}|d w| \leqq L^{p}, \quad p>0
$$

We transform now by the substitution $w=\mathrm{I} / w^{\prime}$; the function $\pi\left(\mathrm{I} / w^{\prime}\right)$ is analytic and different from zero for $\left|w^{\prime}\right|<\mathrm{I}$, and so also is the function $\left[\pi\left(\mathrm{I} / w^{\prime}\right)\right]^{p}$, if we consider a suitable determination of the possibly multiple valued function. Cauchy's formula

yields the inequality

$$
\left[\pi\left(\mathrm{I} / w^{\prime}\right)\right]^{p}=\frac{\mathrm{I}}{2 \pi i} \int_{\gamma} \frac{\left[\pi\left(\mathrm{I} / w^{\prime \prime}\right)\right]^{p} d w^{\prime \prime}}{w^{\prime \prime}-w^{\prime}}
$$

$$
\left|\pi\left(\mathrm{I} / w^{\prime}\right)\right|^{p} \leqq \frac{\mathrm{I}}{2 \pi} \int_{\gamma}\left|\pi\left(\mathrm{I} / w^{\prime \prime}\right)\right|^{p} \frac{\left|d w^{\prime \prime}\right|}{\mathrm{I}-r}, \quad \text { for }\left|w^{\prime}\right| \leqq r<\mathrm{I},
$$

or

$$
\left|\pi\left(\mathrm{I} / w^{\prime}\right)\right|^{p} \leqq \frac{L^{p}}{2 \pi(\mathrm{I}-r)}, \quad\left|w^{\prime}\right| \leqq r<\mathrm{I}
$$

which is the same as

$$
|\pi(w)|^{p} \leqq \frac{L^{p}}{2 \pi(\mathrm{I}-\eta)^{\prime}}, \quad|w| \geqq \frac{\mathrm{I}}{r}>\mathrm{I} .
$$

The function $\left(\mathrm{I}-\bar{\beta}_{i} w\right) /\left(w-\beta_{i}\right)$ has a modulus greater than unity for $|w|<\mathrm{I}$, so this last inequality implies

$$
\left|P[\Psi(w)] \frac{\left(w-\alpha_{1}\right)\left(w-\alpha_{2}\right) \cdots\left(w-\alpha_{n}\right)}{\left(\mathrm{I}-\bar{\alpha}_{1} w\right)\left(\mathrm{I}-\bar{\alpha}_{2} w\right) \cdots\left(\mathrm{I}-\bar{\alpha}_{n} w\right)}\right| \leqq \frac{L}{[2 \pi(\mathrm{I}-r)]^{\frac{1}{p}}}, \quad|w| \geqq \frac{\mathrm{I}}{r}>\mathrm{I} .
$$

It is readily shown that

54-31104. Acta mathematica. 57. Imprimé le 3 septembre 1931. 


$$
\left|\frac{\mathrm{I}-\bar{\alpha}_{i} w}{w-\alpha_{i}}\right| \leqq \frac{R_{1} \varrho-\mathrm{I}}{\varrho-R_{1}}, . \quad \text { for }|w|=R_{1}<\varrho,
$$

and from this inequality Lemma II follows immediately.

Lemma II is in reality more general than Lemma $I$, in the sense that it yields an easy proof of Lemma I, but we shall find it nevertheless convenient to have Lemma $I$ for reference.

Let us now prove Theorem $I$ in case approximation is measured by the method 2). By Theorem II there exists a sequence of rational functions $r_{n}(z)$ of respective degrees $n$ with their poles in the set $E$ such that we have for an arbitrary $R$

$$
\left|f(z)-r_{n}(z)\right| \leqq \frac{M}{R^{n}}, \quad z \text { on } C
$$

where $M$ depends on $R$ but not on $n$. The present measure of approximation of $r_{n}(z)$ to $f(z)$ is

$$
\int_{\gamma} n(w)\left|f(z)-r_{n}(z)\right|^{p}|d w|, \quad p>0,
$$

where $n(w)$ is continuous and positive on $\gamma$. An inequality of the form

$$
\int_{\gamma} n(w)\left|f(z)-v_{n}(z)\right|^{p}|d w| \leqq \frac{M^{\prime}}{R^{n p}}
$$

is satisfied for the particular rational functions $r_{n}(z)$ just mentioned, and so this same inequality holds for the sequence of rational functions $r_{n}(z)$ of best approximation. If we have $o<n^{\prime}<n(w)$ for $w$ on $\gamma$, inequality (4.3) implies

$$
\int_{\gamma}\left|f(z)-r_{n}(z)\right|^{p}|d w| \leqq \frac{M^{\prime}}{n^{\prime} \bar{R}^{n p}} .
$$

We are now in a position to use inequality (4.4) for two successive values of $n$ and to apply the general inequalities

$$
\int\left|\chi_{1}+\chi_{2}\right|^{p} d x \leqq 2^{p-1} \int\left|\chi_{1}\right|^{p} d x+2^{p-1} \int\left|\chi_{2}\right|^{p} d x, \quad p>1
$$

$$
\int\left|\chi_{1}+\chi_{2}\right|^{p} d x \leqq \int\left|\chi_{1}\right|^{p} d x+\int\left|\chi_{2}\right|^{p} d x, \quad 0<p \leqq \mathrm{I} .
$$


There results the inequality

$$
\int_{\gamma}\left|r_{n}(z)-r_{n-1}(z)\right|^{p}|d w| \leqq \frac{M_{1}}{R^{n p}},
$$

where $R$ is arbitrary and $M_{1}$ depends on $R$. Our conclusion follows now from Lemma II by the method used in connection with Lemma $I$.

It will be noted that the function $F(z)$ to which the sequence $r_{n}(z)$ converges must coincide with $f(z)$ on $\gamma$, for the inequality

$$
\int_{\gamma}\left|F(z)-r_{n}(z)\right|^{p}|d w| \leqq \frac{M}{R^{n p}},
$$

which is a consequence of (3.5), yields by (4.4) and (4. 5)

$$
\int_{\gamma}|F(z)-f(z)|^{p}|d w| \leqq \frac{N}{R^{n p}} .
$$

Hence the integral on the left is zero and the functions $F(z)$ and $f(z)$ coincide almost everywhere on $\gamma$. Thus $F(z)$ and $f(z)$ coincide at an infinity of points of $C$ and are identical.

5. Approximation measured by a Line Integral. Let us now turn to method 3) as a measure of approximation, namely that $C$ is an arbitrary closed limited point set whose boundary is a rectifiable Jordan are or curve or other point set of positive linear measure, and whose complement (i. e. of $C$ ) is simply connected; approximation is measured in the sense of weighted $p$-th powers $(p>0)$ by a line integral over $C$. In particular $C$ may be a region bounded by a rectifiable Jordan curve - in this case the proof of Theorem I is especially simple - or may be composed of even a suitable infinity of such regions, together with Jordan ares abutting on and exterior to them. This measure of approximation (for $p=2$ ) has been used by Szegö for approximation of given functions by polynomials ${ }^{1}$ in case $C$ is either a Jordan curve or are.

We shall need the following lemma:

Lemma III. Let $C$ be an arbitrary closed limited point set whose boundary $C^{\prime}$ has positive linear measure, whose complement is simply connected, and denote by $w=\boldsymbol{\Phi}(z), z=\Psi(w)$ a function which maps the complement of $C$ onto the exterior

${ }^{1}$ See particularly Mathematische Zeitschrift, vol. 9 (I92I), pp. 218-270. 
of the unit circle $\gamma$ in the w-plane so that the points at infinity correspond to each other. Let $C_{R}$ denote the curve $|\Phi(z)|=R>_{\mathrm{I}}$ in the z-plane. If $P(z)$ is a rational function of degree $n$ whose poles lie exterior to $C_{\rho}, \varrho>1$, and if we have

$$
\int_{C^{\prime}}|P(z)|^{p}|d z| \leqq L^{p}, \quad p>0,
$$

then we have likewise

$$
|P(z)| \leqq L L^{\prime}\left(\frac{\varrho R_{1}-\mathrm{I}}{\varrho-R_{1}}\right)^{n}, \quad z \text { on } C_{R_{1}}, \quad R_{1}<\varrho,
$$

where $L^{\prime}$ depends on $R_{1}$ but not on $P(z)$.

The boundary $C^{\prime}$ is composed of a connected set consisting of a finite or infinite number of Jordan curves and arcs, and we shall need later to consider the plane cut along $C^{\prime}$. For the truth of Lemma III and of Theorem I in case approximation is measured by 3 ), it is immaterial whether in such an integral as (5. 1) [or (5.3)] we consider the cut plane or uncut plane; in the cut plane Jordan arcs belonging to $C^{\prime}$ not parts of Jordan curves belonging to $C^{\prime}$ are naturally to be counted twice in the integral. However, we shall later use Cauchy's integral formula for the region $D$ complementary to $C$. If an integral is extended over a curve $K$ in $D$ and if $K$ varies monotonically so that every point of $D$ is exterior to some position of $K$, then $K$ approaches as a limiting position the point set $C^{\prime}$, where the plane is cut along $C^{\prime}$ - that is, where each are of $C^{\prime}$ not part of the boundary of a region belonging to $C$ is counted doubly. As a matter of convenience, then, we shall suppose that in considering integrals over $C^{\prime}$, each are of $C^{\prime}$ not part of the boundary of a region belonging to $C$ is counted doubly. The weight function $n(z)$ used below may, if desired, be considered to have two distinct values at points of such an arc $C^{\prime}$, corresponding to the double valence.

The function $\boldsymbol{\Phi}(z)$ is continuous in the $z$-plane cut along the point set $C^{\prime}$. Let the poles and zeros of $P(z)$ on the complement of $C$ be respectively $\alpha_{1}, \alpha_{2}, \ldots, \alpha_{n}$ and $\beta_{1}, \beta_{2}, \ldots, \beta_{n}$. We may have less than $n$ zeros or poles or both, but that requires only a slight and obvious modification in the reasoning now to be used. The function

$$
\begin{aligned}
\pi(z)=P(z) \frac{\boldsymbol{\Phi}(z)-\boldsymbol{\Phi}\left(\alpha_{1}\right)}{\mathrm{I}-\overline{\boldsymbol{\Phi}}\left(\alpha_{1}\right) \boldsymbol{\Phi}(z)} \frac{\boldsymbol{\Phi}(z)-\boldsymbol{\Phi}\left(\alpha_{2}\right)}{\mathrm{I}-\overline{\boldsymbol{\Phi}}\left(\alpha_{2}\right) \boldsymbol{\Phi}(z)} \cdots \frac{\boldsymbol{\Phi}(z)-\boldsymbol{\Phi}\left(\alpha_{n}\right)}{\mathrm{I}-\overline{\boldsymbol{\Phi}}\left(\alpha_{n}\right) \boldsymbol{\Phi}(z)} \\
\frac{\mathrm{I}-\overline{\boldsymbol{\Phi}}\left(\beta_{1}\right) \boldsymbol{\Phi}(z)}{\boldsymbol{\Phi}(z)-\boldsymbol{\Phi}\left(\beta_{1}\right)} \frac{\mathrm{I}-\overline{\boldsymbol{\Phi}}\left(\beta_{2}\right) \boldsymbol{\Phi}(z)}{\boldsymbol{\Phi}(z)-\boldsymbol{\Phi}\left(\beta_{2}\right)} \cdots \frac{\mathrm{I}-\overline{\boldsymbol{\Phi}}\left(\beta_{n}\right) \boldsymbol{\Phi}(z)}{\boldsymbol{\Phi}(z)-\overline{\boldsymbol{\Phi}}\left(\beta_{n}\right)}
\end{aligned}
$$


is analytic and different from zero on the complement $D$ of $C$, and so therefore is $[\pi(z)]^{p}$. On $C^{\prime}$, the two functions $\pi(z)$ and $P(z)$ have the same modulus.

The function $[\pi(z)]^{p / \Phi}(z)$ is analytic at infinity and vanishes there, so we have

$$
\frac{[\pi(z)]^{p}}{\bar{\Phi}(z)}=\frac{\mathrm{I}}{2 \pi i} \int_{C^{\prime}} \frac{[\pi(t)]^{p}}{\Phi(t)} \frac{d t}{t-z}, z \text { in } D
$$

$$
\left|\frac{[z c(z)]^{p}}{\Phi(z)}\right| \leqq \frac{L^{p}}{2 \pi \delta}, \quad z \text { on } C_{R_{1}}
$$

where $\delta$ is suitably chosen. The integral over $C^{\prime}$ is the ordinary integral, in the positive direction with respect to $D$.

The function $\left[\mathrm{I}-\bar{\Phi}\left(\beta_{i}\right) \Phi(z)\right] /\left[\Phi(z)-\Phi\left(\beta_{i}\right)\right]$ has a modulus greater than unity for $z$ on $C_{R_{1}}$, and for $z$ on $C_{R_{1}}$ the function $\left[\Phi(z)-\Phi\left(\alpha_{i}\right)\right] /\left[\mathrm{I}-\bar{\Phi}\left(\alpha_{i}\right) \Phi(z)\right]$ has a modulus not less than $\left(\varrho-R_{1}\right) /\left(\varrho R_{1}-\mathrm{I}\right)$, so Lemma III follows at once.

The method of application of Lemma III to the proof of Theorem I is quite similar to that of Lemma II. By Theorem II there exists some sequence of rational functions $r_{n}(z)$ of respective degrees $n$ with their poles in the set $E$ such that we have for an arbitrary $R$

$$
\left|f(z)-r_{n}(z)\right| \leqq \frac{M}{R^{n}}, \quad z \text { on } C .
$$

Our present measure of approximation is

$$
\int_{C^{\prime}} n(z)\left|f(z)-r_{n}(z)\right|^{p}|d z|, \quad p>0,
$$

where $n(z)$ is continuous and positive on $C^{\prime}$. An inequality of the form

$$
\int_{C^{\prime}} n(z)\left|f(z)-r_{n}(z)\right|^{p}|d z| \leqq \frac{M^{\prime}}{R^{n p}}
$$

is satisfied for this particular set of rational functions $r_{n}(z)$ and so the same inequality holds for the sequence of rational functions of best approximation. If we have $0<n^{\prime}<n(z)$ for $z$ on $C^{\prime}$, inequality (5.3) implies

$$
\int_{C^{\prime}}\left|f(z)-r_{n}(z)\right|^{p}|d z| \leqq \frac{M^{\prime}}{n^{\prime} R^{n p}},
$$


which, used for two succesive values of $n$, implies by the use of inequality (4. 5)

$$
\int_{C^{\prime}}\left|r_{n}(z)-r_{n-1}(z)\right|^{p}|d z| \leqq \frac{M_{1}}{R^{n p}} .
$$

This inequality is of the precise form for application of Lemma III, and by the methods already used yields Theorem $I$ for the measure of approximation which we have been considering.

6. Approximation in a Region; Conformal Mapping. Method 4) of measuring approximation is next to be studied, namely that $C$ is an arbitrary simply connected region and approximation is measured in the sense of weighted $p$-th powers $(p>0)$ by integration over the circle $\gamma:|w|=\mathbf{I}$ when the interior of $C$ is mapped conformally onto the interior of $\gamma$. This method (without the use of a weight function and for $p>\mathrm{I}$ ) has recently been used by $\mathrm{Julia}^{1}$ in the study of approximation of harmonic functions by harmonic polynomials.

If we are dealing with either of the measures of approximation 4) or 5), lemmas precisely analogous to those already established may be used, but it is just as convenient to proceed in a somewhat different way. Let us prove ${ }^{2}$

Lemma IV. If each of the functions $P_{n}(z)$ is analytic and bounded interior to the simply connected region $C$ and if we have

$$
\int_{\gamma}\left|P_{n}(z)\right|^{p}|d w| \leqq L^{p}, \quad p>0,
$$

where the interior of $C$ is mapped onto the interior of $\gamma:|w|=I$, then we have

$$
\left|P_{n}(z)\right| \leqq L^{\prime} L,
$$

for $z$ on an arbitrary elosed point set $C^{\prime}$ interior to $C$, where $L^{\prime}$ depends on $C^{\prime}$ but not on $P_{n}(z)$.

In the integral in (6. I) the value of $\left|P_{n}(z)\right|$ on $\gamma$ is naturally to be taken in the sense of normal approach to $\gamma$; these boundary values are known to exist.

Let the zeros of $P_{n}(z)$, if any, interior to $C$ be $\alpha_{1}, \alpha_{2}, \ldots$ We assume $P_{n}(z)$ not identically zero, for the lemma is obviously true so far as concerns such functions. Consider the function

1 Acta Litterarum ac Scientiarum (Szeged), vol. 4(1929), pp. $217-226$.

2 Compare Walsh, Transactions of the American Mathematical Society, vol. 33 (I93I), pp. $370-388$. 


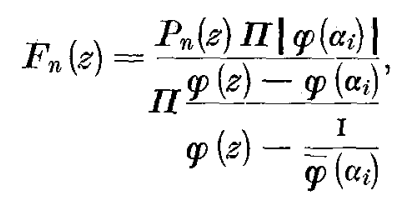

where $w=\rho(z), z=\psi(w)$ is a function which maps the interior of $C$ conformally onto the interior of $\gamma$. There may be an infinity of points $\alpha_{i}$ but if so the infinite products here converge, by Blaschke's theorem. We assume $\varphi\left(\alpha_{i}\right) \neq 0$, which involves no loss of generality, for the following reasoning concerning $P_{n}(z)$ may be applied to the quotient by $[\varphi(z)]^{k}$ of a given $P_{n}(z)$, where $k$ is the order of the zero of the given $P_{n}(z)$ at the point $z=\psi(0)$. The function $F_{n}(z)$ is analytic and different from zero interior to $C$, and has the same modulus as $P_{n}(z)$ on $C$ or on $\gamma$. The function $\left[F_{n}(z)\right]^{p}$ is likewise analytic and uniformly bounded interior to $C$ and $\gamma$, if we consider an arbitrary determination of the $p$-th power at an arbitrary point interior to $C$ or $\gamma$ and is analytic extension, so we have Cauchy's integral

$$
\left\{F_{n}[\psi(w)]\right\}^{p}=\frac{1}{2 \pi i} \int_{\gamma}\left\{F_{n}[\psi(t)]\right\}^{p} \frac{d t}{t-w}
$$

Cauchy's integral is naturally valid here, for the boundary values of $\psi(w)$ and hence of $F_{n}(z)$ for normal approach to $\gamma$ exist almost everywhere.

It follows now that we have

$$
\left|F_{n}(z)\right|^{p} \leqq \frac{\mathrm{I}}{2 \pi(\mathrm{I}-r)} \int_{\gamma}\left|F_{n}(z)\right|^{p}|d w| \leqq \frac{L^{p}}{2 \pi(\mathrm{I}-r)}, \quad|\varphi(z)| \leqq r<\mathrm{I} .
$$

Each function

$$
\frac{\left|\varphi\left(\alpha_{i}\right)\right|}{\frac{\varphi(z)-\varphi\left(\alpha_{i}\right)}{\varphi(z)-\frac{1}{\bar{\varphi}\left(\alpha_{i}\right)}}}
$$

is of absolute value greater than unity for $z$ interior to $C$, so we have from $\left(\begin{array}{ll}6 & 2\end{array}\right)$

$$
\left|P_{n}(z)\right|^{p} \leqq\left|F_{n}(z)\right|^{p} \leqq \frac{L^{p}}{2 \pi(\mathrm{I}-r)}, \quad|\varphi(z)| \leqq r<\mathrm{I},
$$

and the proof of the lemma is complete. 
The application of Lemma IV is immediate. By Theorem III there exist rational functions $r_{n}(z)$ of respective degrees $n$ with poles on the set $E$ such that we have

$$
\left|f(z)-r_{n}(z)\right| \leqq \frac{M}{R^{n}}, \quad z \text { on } C
$$

so the inequality

$$
\int_{\gamma} n(w)\left|f(z)-r_{n}(z)\right|^{p}|d w| \leqq \frac{M^{\prime}}{R^{n p}}
$$

where the weight function $n(w)$ is positive and continuous on $\gamma$, is satisfied for this particular set of rational functions and hence for the rational functions of best approximation. This leads in turn to inequalities of the form

$$
\int_{\gamma}\left|f(z)-r_{n}(z)\right|^{p}|d w| \leqq \frac{M^{\prime}}{n^{\prime} R^{n p}} .
$$

This last inequality yields by Lemma IV

$$
\left|f(z)-r_{n}(z)\right| \leqq \frac{M_{z}}{R^{n}}, \quad z \text { on } C^{\prime}
$$

where $C^{\prime}$ is an arbitrary closed point set interior to $C$ and where $R$ is arbitrary, whence Theorem I follows by Theorem III for the measure of approximation that we are here considering.

7. Approximation in a Region; Surface Integrals. Method 5) of measiuring approximation involves the use of a double integral,

$$
\iint_{C} n(z)\left|f(z)-r_{n}(z)\right|^{p} d S, \quad p>0
$$

and this method has been used by Carleman ${ }^{1}$ in considering the approximation to an analytic function by polynomials. We shall find it convenient to prove

Lemma V. If each of the functions $P_{n}(z)$ is analytic interior to an arbitrary region $C$, and if we have

$$
\iint_{C}\left|P_{n}(z)\right|^{p} d S \leqq L^{p} \quad p>0
$$

\footnotetext{
${ }^{1}$ Arkiv för Matematik, Astronomi och Fysik, vol. 17 (1922-23).
} 
then we have

$$
\left|P_{n}(z)\right| \leqq L^{\prime} L
$$

for $z$ on an arbitrary closed point set $C^{\prime}$ interior to $C$, where $L^{\prime}$ depends on $C^{\prime}$ but not on $P_{n}(z)$.

The integral

$$
\frac{\mathrm{I}}{2 \pi} \int_{0}^{2 \pi}\left|P_{n}\left(z_{0}+r e^{i \theta}\right)\right|^{p} d \theta, \quad p>0
$$

is well known to be a non-decreasing function of $r$, in an arbitrary circle $K$ which together with its interior lies interior to $C$. Here $(r, \theta)$ are polar coordinates with pole at the point $z_{0}$. The limit of this integral as $r$ approaches zero is obviously $\left|P_{n}\left(z_{0}\right)\right|^{p}$, from which follows the inequality

$$
\left|P_{n}\left(z_{0}\right)\right|^{p} \leqq \frac{1}{2 \pi} \int_{0}^{2 \pi}\left|P_{n}\left(z_{0}+r e^{i \theta}\right)\right|^{p} d \theta
$$

We multiply both members of this inequality by $r d r$ and integrate from zero to $k$, the radius of $K$. The resulting inequality is

$$
\frac{k^{2}}{2}\left|P_{n}\left(z_{0}\right)\right|^{p} \leqq \frac{\mathrm{I}}{2 \pi} \iint_{K}\left|P_{n}(z)\right|^{p} d S
$$

so we may write by virtue of $(7.1)$

$$
\left|P_{n}\left(z_{0}\right)\right|^{p} \leqq \frac{\mathrm{I}}{\pi k^{2}} \int_{K} \int_{K}\left|P_{n}(z)\right|^{p} d S \leqq \frac{\mathrm{I}}{\pi k^{2}} \iint_{C}\left|P_{n}(z)\right|^{p} d S \leqq \frac{L^{p}}{\pi k^{2}}
$$

This inequality holds for every point $z_{0}$ interior to $C$ provided merely that the distance from $z_{0}$ to the boundary of $C$ is not less than $k$. The inequality therefore holds for proper choice of $k$ for $z_{0}$ on an arbitrary closed point set $C^{\prime}$ interior to $C$ and is equivalent to $(7.2)$ for $z$ on $C^{\prime}$, so the lemma is completely established.

The application of Lemma $\mathrm{V}$ in the proof of Theorem $\mathrm{I}$ does not differ materially from the application of Lemma IV and is left to the reader. Theorem $I$ is now completely proved.

55-31104. Acta mathematica. 57. Imprimé le 4 septembre 1931. 
8. Further Remarks. There are three problems, distinct from those already treated, which are intimately connected with the discussion given. We mention merely the statement of the problems and leave the details to the reader. In each of the three cases some new results can be found directly from our previous work, while other new results lie but little deeper.

I. The given function $f(z)$ may be meromorphic instead of analytic on $O$ and the approximating rational functions $r_{n}(z)$ of respective degrees $n$ are permitted to have poles in all the singularities $E$ of $f(z)$, in particular in the poles of $f(z)$ belonging to $C$. Under certain conditions it is still true that the sequence of rational functions of best approximation whose poles lie in the singularities of $f(z)$, converges to the limit $f(z)$ on the entire plane except at the singularities of $f(z){ }^{1}$

2. The given function $f(z)$ may be analytic or meromorphic on $C$ and the given rational functions $r_{n}(z)$ may be required or not to satisfy auxiliary conditions interior to $C$, those conditions being the prescription of the values of $r_{n}(z)$ with perhaps some of its derivatives at various points interior to $C$; indeed, the functions $r_{n}(z)$ may be allowed to be meromorphic interior to $C$, and have their principal parts prescribed at various points interior to $C$. These auxiliary conditions need have no relation to the given function $f(z)$. If the auxiliary conditions do not depend on $n$, if the limit function $F(z)$ (which is uniquely determined by $f(z)$ and the auxiliary conditions) of the sequence $r_{n}(z)$ has all of its singularities in a point set $E$ one of whose derivatives is empty, and if the poles of the approximating functions $r_{n}(z)$ are merely restricted to lie on $E$, then under suitable simple restrictions on $C$, the sequence of rational functions $r_{n}(z)$ of best approximation to $f(z)$ on the boundary of $C$ in the sense of Tchebycheff and satisfying the auxiliary conditions, converges to the function $F(z)$ on the entire plane except at the singularities of $F(z){ }^{2}$ If the prescribed auxiliary conditions involve merely the coincidence of the values of $r_{n}(z)$ and the given function $f(z)$ at certain points interior to $C$, then under suitable conditions we have the conclusion of Theorem I satisfied: the sequence $r_{n}(z)$ approaches the function $f(z)$ at every point of the plane not on $E$, uniformly on any closed

\footnotetext{
${ }^{1}$ Compare Walsh, Transactions of the American Mathematical Society, vol. 30 (1928), pp. $838-847$.

${ }^{2}$ Compare Walsh, Transactions of the American Mathematical Society, vol. 32 (1930), pp. $335-390$.
} 
point set containing no point of $E$, where approximation is measured by any of the methods I) -5 ).

3. The results of the present paper have application to the study of approximation of harmonic functions by harmonic rational functions. If a suitably restricted harmonic function $u(x, y)$ is given, the function

$$
f(z)=u(x, y)+\ddot{v} v(x, y),
$$

where $v(x, y)$ is a function conjugate to $u(x, y)$, satisfies the hypothesis of Theorem I. Approximation to $f(z)$ by rational functions $r_{n}(z)=r_{n}^{\prime}(x, y)+i r_{n}^{\prime \prime}(x, y)$ implies approximation to $u(x, y)$ by the harmonic rational functions $r_{n}(x, y)$. Even if the given function $u(x, y)$ is not so simple that an equation of form (8. I) is valid, where $f(z)$ satisfies the hypothesis of Theorem $\mathrm{I}$, it may be possible to approximate $u(x, y)$ by harmonic rational functions plus harmonic functions involving the logarithms of distances. Such methods of approximation have already been used to some extent by the present writer. ${ }^{1}$

\footnotetext{
1 Bulletin of the American Mathematical Society, vol. 35 (1929), pp. 499-544.
} 